\title{
Clinical application of CyberKnife for high-risk central nervous system tumors: A clinical trial report of 60 cases
}

\author{
XIN WANG, YUAN-YUAN WANG, PENG JIANG, JIAN-JUN MA, ZHEN QU, \\ HAN-CHEN LIU, SHAN-SHAN WANG and YI-SHAN WANG
}

Center for Non-Traumatic Treatment and Diagnosis of Tumors, The People's Liberation Army 107th Hospital, Affiliated Hospital of Bin Zhou Medical College, Shandong 264002, P.R. China

Received August 12, 2011; Accepted September 13, 2011

DOI: $10.3892 /$ etm.2011.356

\begin{abstract}
The objective of the present study was to evaluate the application potential of CyberKnife for high-risk tumors of the central nervous system and to analyze the effectiveness of CyberKnife in relation to dose recovery and gain division (times). A total of Eighty-one targeted areas from 139 central nervous tumor lesions in 60 patients were treated with I-VI ranged CyberKnife for 1 week. Following CyberKnife treatment, imaging tests revealed a decrease in tumor volume, reduction of central nervous system symptoms and an increase in the life quality of patients. The advantages of CyberKnife include non-invasiveness, individualized treatment, flexibility, high accuracy and rapid treatment. CyberKnife produces slight damage and a favorable therapeutic effect and expands our concepts concerning precise radiotherapy for tumors. It is an indispensable method for personalized tumor treatment.
\end{abstract}

\section{Introduction}

Patients with high-risk central nervous system tumors commonly refuse surgery or receive partial resection due to the difficulty and risk of surgery, and the rapid recurrence of tumors (1-3). Palliative treatment is often applied because of the inability to perform surgery, regular radiation or chemotherapy due to the invasion of central organs with important functions by tumor infiltration and multiple metastases (4-6). Direct involvement or activation of local nociceptors as well as adjacent nerves, vessels, central nervous tissue as suppressed by tumors may lead to paralysis or paraplegia accompanied by pain and tumor compression complex (7-9). Using CyberKnife to control or reduce local lesions, we attempted to improve the

Correspondence to: Professor Yi-Shan Wang, Center for Non-Traumatic Treatment and Diagnosis of Tumors, The People's Liberation Army 107th Hospital, Affiliated Hospital of Bin Zhou Medical College, No. 7 Southern Zhichu Road, Yantai, Shandong 264002, P.R. China

E-mail: wangyishan288@163.com

Key words: central nervous system, high-risk tumor, CyberKnife, radiotherapy, dose response internal environment, to reduce adverse reactions, to control tumor progression and to alleviate clinical symptoms.

\section{Materials and methods}

Subjects. A total of 139 central nervous system tumor lesions in 60 patients who received palliative treatment at the Center for Non-Traumatic Treatment and Diagnosis of Tumor, Binzhou Medical College Affiliated with the PLA 107th Hospital, from October 2010 to May 2011, were selected for the study according to the following standards. i) Diagnosis of tumors was confirmed by pathology or imaging tests, such as CT and MRI, and were ranked as stages III-IV according to the WHO clinical staging system. ii) Patients had an ECOG Performance status 2-4. iii) Patients exhibited crudescence following surgery, invasion of central organs with vital functions, infiltration of lymph and neurons or multiple metastases and had no indications for surgery or were unable to undergo standard chemical or radiotherapy. iv) Patients provided informed consent. The exclusion criteria included i) associated $\mathrm{Tb}$, undetermined diagnosis, early stage disease with surgical indications; ii) intractable increased intracranial pressure; iii) recent repeated radiotherapy or suspected radiotherapy complications.

A total of 38 males and 22 females, 6-63 years of age, with an average age of 50 years, were enrolled. There were 81 targeted areas from 139 lesions including 9 gliomas, 23 brain metastases, 8 meningiomas, 11 pituitary tumors, 8 intramedullary spinal cord tumors and 1 spinal meningioma. Between 1 and 24 lesions were noted in each patient, 1-6 lesions were planned to be targeted, and 31 cases suffered from brain swelling.

Methods. CT slices (1.25-mm) were performed after hospitalization. IMR, PET-CT or DSA image fusion was conducted when necessary to determine the target areas. Centrum or skull 6D tracking outlined target CTV. Treatment plans and treatment doses were determined according to the number of tumor lesions and tumor size. Treatment was carried out 1-6 times, and complete treatment spanned 1 week.

Evaluation. Stereotactic radiowave surgery platform specifications: CyberKnife System (Accuray Group Co., Ltd., USA). The 
A

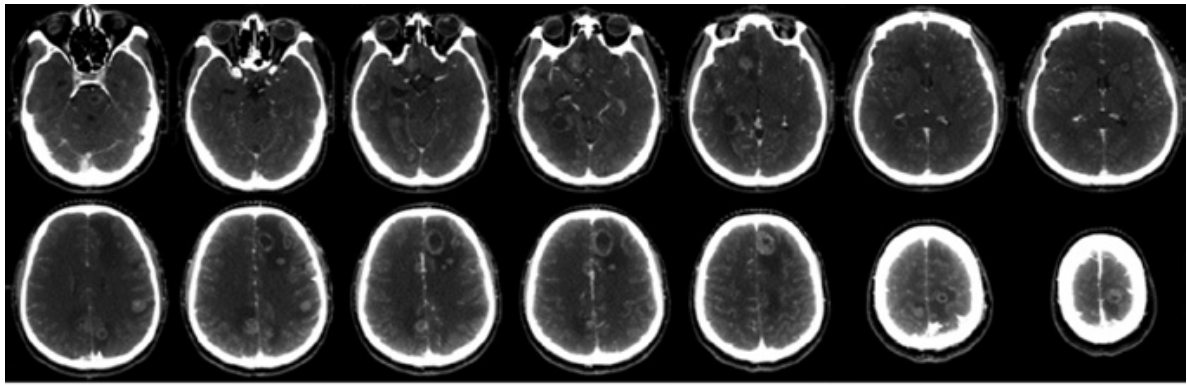

B

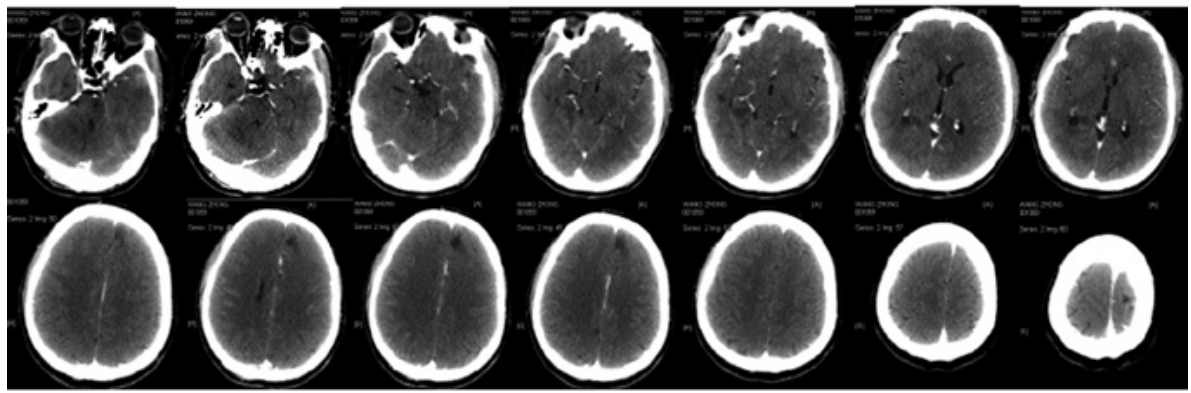

Figure 1. Multiple intracranial metastases (24 lesions). CT revealed that tumors generally disappeared after CyberKnife treatment. (A) CT before CyberKnife therapy (2010-12-04); (B) CT after CyberKnife therapy (2011-02-22).

output dose rate was 400-600 cGy. The US FDA granted market access for systemic treatment as certified in August 2001. Tumor dose coverage was $85-95 \%$. For patients with small and single-tumor lesions, the general dose was 1-3 F; for those with large and multiple tumor lesions the general dose was 4-6 F. The tumor dose DT was 18-60 Gy, with a single dose of 4-18 Gy.

Criteria. Evaluation: i) imaging examination, shrinkage; ii) RIA and endocrine hormone detection, decrease or recover; iii) Zubrod-ECOG-WHO (ZPS) (10) scale 1-4: 0, normal activity; 1, some symptoms but still almost fully ambulatory; $2,<0 \%$ of daytime spent in bed; $3,>50 \%$ of daytime spent in bed; 4 , completely bedridden; 5 , deceased.

WHO objective criteria of curative effect (11). CR, complete remission with symptoms and physical signs totally disappeared for 4 weeks; PR, partial remission with tumor volume shrinkage $>50 \%$ for at least 4 weeks; NC, no significant change observable for at least 4 weeks with a tumor volume increase of $\leq 25 \%$ or shrinkage $\geq 50 \%$; PD, progressive disease with new lesions appearing or the original lesion increasing $>25 \%$ in size. The total effective rate was calculated with the following equation: $(\mathrm{CR}+\mathrm{PR}) /$ total cases $\mathrm{x} 100 \%$.

Statistical analysis. All values are expressed as the means \pm standard deviation (SD). Differences were evaluated using Statistical Package for Social Science 13.0 (SPSS13.0). Statistical analysis was performed using the two-sided Student's t-test. Differences were considered statistically significant at the level of $\mathrm{p}<0.05$.

\section{Results}

Case statistics. A total of 63 cases were assessed. Three cases were excluded; 2 cases could not follow the study instructions because of age and 1 case experienced financial problems. A total of 60 cases were involved in the final analysis.
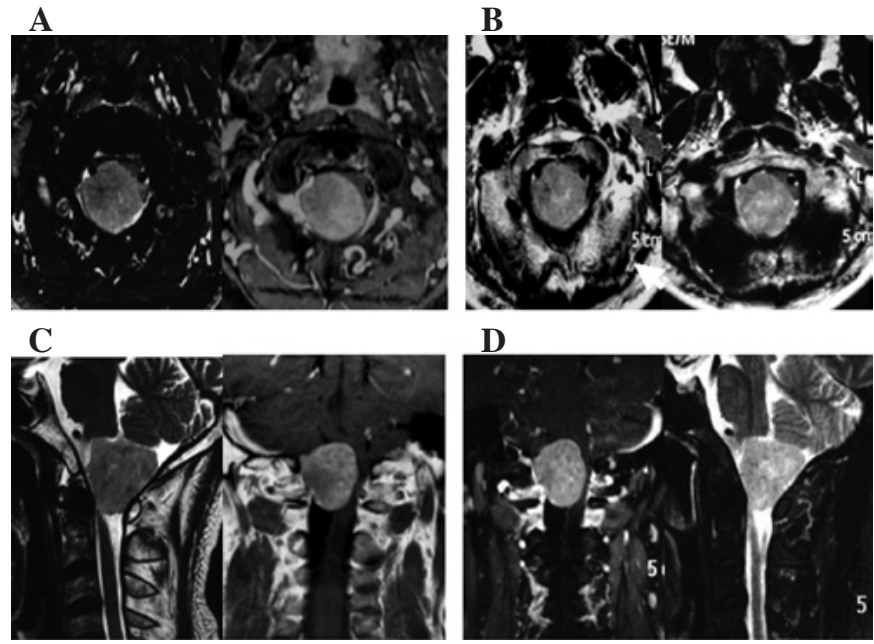

Figure 2. Meningioma $(3.4 \times 3 \times 2.7 \mathrm{~cm})$. Although there was no significant change in radiographic imaging, the patient experienced improvement in the quality of life after CyberKnife therapy. (A) MRI before CyberKnife therapy (2011-02-25); (B) MRI after CyberKnife therapy (2011-04-25); (C) MRI before CyberKnife therapy (2011-02-25); (D) MRI after CyberKnife therapy (2011-04-25).

Imaging tests. High-risk patients with at least one of the following conditions (tumor recurrence after surgery, multiple metastases, disease uncontrollable by either radiation or chemotherapy, invalid conservative therapy, or diagnosis at an advanced stage) exhibited significant shrinkage or disappearance of lesions as confirmed by imaging compared to 120 advanced patients who received only conservative therapy by conformal radiation therapy.

RIA monitoring and endocrine hormone condition. Compared to the control group, 5 of 24 patients with increased tumor markers or endocrine hormone became negative, 10 cases were degraded to varying degrees, 6 cases had no obvious changes and 3 cases increased following CyberKnife treatment. 
A

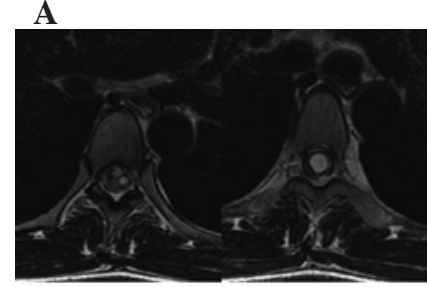

C

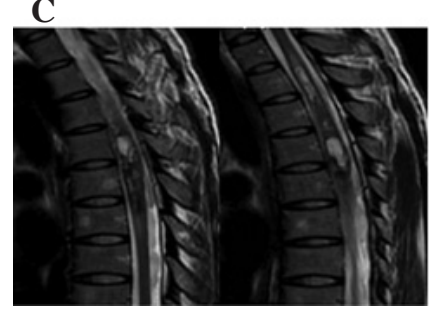

B

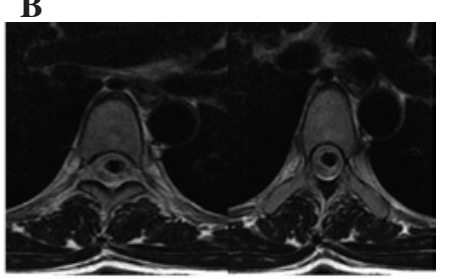

D

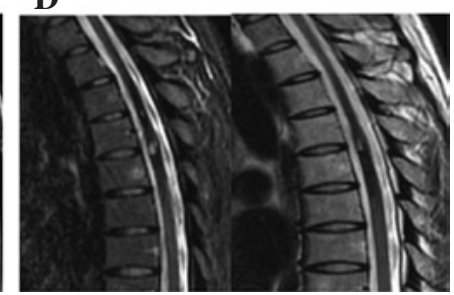

Figure 3. A patient with chest intramedullary hemangioma $(14 \mathrm{~cm})$ was able to walk alone after he was treated in four fractions. (A) CT before CyberKnife therapy (2011-01-30); (B) CT after CyberKnife therapy (2011-04-30); (C) MRI before CyberKnife therapy (2011-01-30); (D) MRI after CyberKnife therapy (2011-04-30).

ZPS scores were improved to various degrees in all the patients after treatment, accompanied by alleviation or disappearance of tumor suppression syndrome and pain syndrome.

Objective effect assessment. Patients with advanced cancer who received repeated treatment also showed a high rate of alleviation of symptoms after CyberKnife treatment. The brain stem invaded by intracranial multiple metastases (24 lesions) caused severe central nervous system suppression syndrome, and the treatment plans were divided into six parts with at most one target for six tumor lesions. The patients were able to take care of themselves after completion of treatment in 19 days (Fig. 1). Patients with meningiomas $\left(3.4 \times 3.0 \times 2.7 \mathrm{~cm}^{3}\right)$ who refused surgery due to the high risk by severe medulla oblongata placeholder successfully accomplished therapeutic treatment utilizing CyberKnife (Fig. 2). Another case with chest intramedullary hemangiomas (reaching a length of $14 \mathrm{~cm}$ ) who was paralyzed in bed and could not care of himself recovered after CyberKnife treatment and was able to walk following 4 administrations of CyberKnife treatment (Fig. 3).

\section{Discussion}

Palliative treatment is the major treatment for patients suffering from central nervous system tumors, particularly brain glioma, metastatic tumors and spinal cord tumors (12-18). Surgery or conventional radiotherapy is unable to provide a complete cure due to the late diagnosis of these advanced tumors, the rapid growth of the tumors, and compression or infiltration of important organs $(13,19-22)$. CyberKnife is a flexible method having the advantage of high accuracy, timely tracking, shorter treatment times, a high-dose, short treatment course, easy patient access to treatment, improved local cure rate and favorable results $(22,23)$. Statistical results from 60 patients with advanced complex tumors and from 120 patients receiving advanced palliative radiotherapy showed that, after treatment, in some cases CyberKnife even achieved complete remission; the effective rate was $71.7 \%$, suggesting an effective noninvasive treatment for cancer.

CyberKnife technology facilitates cancer treatment and improves the local control rate, especially in high-risk patients (24-27). Most patients receiving CyberKnife do not require hospitalization (28-31), treatment time is short $(32,33)$, and no toxic side effects occur (34-36). We believe that treatment time, frequency and dose should be based on the number of tumors, size, severity of the disease, repeated during the length of the treatment time. This means that treatment should reflect the individual needs and not blindly pursue a rapid treatment. CyberKnife can be carried out without hospitalization or early discharge. At the end of treatment, normal routine blood examination should be carried out. However, after 1-2 weeks of treatment, if grain dump, brain edema, intracranial pressure and orthostatic hypotension occurs, it is necessary for the patients to be hospitalized for observation, consolidation effect, thus they can be excluded from CyberKnife 'surgery' which may cause potential adverse reactions (37).

CyberKnife also incorporates the merits of the Gamma (X) knife while overcoming its shortcomings, such as the need for tumor shape and oversized target areas (38). It works effectively in the cancer therapy of central nervous system tumors. CyberKnife could interface with a variety of imaging examinations, including CT, MRI, PET-CT and DSA, and obtain three-dimensional image to offer the most direct evidence for gross targeted region delineation (39). CyberKnife requires more strict target delineation than ordinary radiotherapy, but not as traditional as conformal radiation therapy (40). Careful forethought should be given due to the CyberKnife portrait of fewer but larger doses.

In conclusion, using CyberKnife therapy, the quality of life of patients with high-risk central nervous system cancer markedly improves, symptoms are rapidly alleviated and patients need not undergo surgery (41). CyberKnife is a new effective therapeutic method for treating advanced high-risk central nervous system cancers.

\section{Acknowledgements}

This study was supported, in part, by grants from the National Special Issue of the Ministry of Health of China (no. 2009BX042), the Foundation of Military Scientific and Technological Issue (no. 06G034), and the Project of Key Program of Jinan Military Region (no. 02Z07).

\section{References}

1. Thariat J, Bolle S, Demizu Y, et al: New techniques in radiation therapy for head and neck cancer: IMRT, CyberKnife, protons, and carbon ions, Improved effectiveness and safety? Impact on survival? Anticancer Drugs 21: 3, 2010.

2. Qiao X, Tullgren O, Lax I, et al: The role of radiotherapy in treatment of stage I non-small cell lung cancer. Lung Cancer 41: $1-11,2003$.

3. McGarry RC, Papiez L, Williams M, et al: Stereotactic body radiation therapy of early-stage non-small-cell lung carcinoma: phase I study. Int J Radiat Oncol Biol Phys 63: 1010-1015, 2005.

4. Wulf J, Haedinger U, Oppitz U, et al: Stereotactic radiotherapy for primary lung cancer and pulmonary metastases: a noninvasive treatment approach in medically inoperable patients. Int $\mathrm{J}$ Radiat Oncol Biol Phys 60: 186-196, 2004. 
5. Yamazaki H, Shiomi H, Tsubokura T, et al: Quantitative assessment of inter-observer variability in target volume delineation on stereotactic radiotherapy treatment for pituitary adenoma and mentingioma optic tract. Radiat Oncol 6: 10, 2011.

6. Hasney CP, Swanton RG and Friendlander PL: CyberKnife stereotactic radiosurgery for recurrent squamous cell carcinoma of the head and neck following salvage surgery with close or positive margins. Laryngoscope 4: 152, 2010.

7. Wang YS, Wang QW, Jia XF, et al: Thermal dose effect of regional radiofrequency hyperthermia on metaphase and advanced stage tumor. J Clin Rehab Tissue Engineering Res 11: 9011-9015, 2007.

8. Dewas S, Dewas-Vautravers C, Servent V, et al: Results and special considerations when treating elderly patients with CyberKnife: a review of 345 cases. Crit Rev Oncol Hematol 76: 1-7, 2010.

9. Xie Y, Djajaputra D, King CR, et al: Intrafractional motion of the prostate during hypofractionated radiotherapy. Int J Radiat Oncol Biol Phys 72: 236-246, 2008.

10. Unger F, Dominikus K and Haselsberger K: [Stereotactic radiosurgery and fractionated stereotactic radiotherapy of acoustic neuromas.] HNO 59: 31-37, 2011 (In German).

11. Sharma S, Ott J, Williams J, et al: Dose calculation accuracy of the Monte Carlo Algorithm for CyberKnife compared with other commercially available dose calculation algorithms. Med Dosim 35: 1-4, 2010.

12. Wang YS, Xu XD and Wang QW: Clinical research of partial IMCRT treated malignant ascites combined with Chinese medicine RF. Chin Med Front 18: 85-87, 2007.

13. Marcial VA, Pajak TF, Kramer S, et al: Radiation Therapy Oncology Group (RTOG) studies in head and neck cancer. Sem Oncol 15: 39-60, 1988.

14. Jereczed-Fossa BA, Beltramo G, Fariselli L, et al: Robotic image-guided stereotactic radiotherapy, for isolated recurrent primary, lymph node or metastatic prostate cancer. Int J Radiat Oncol Biol Phys 79: 1-9, 2011.

15. Degen JW, Gagnon GJ, Voyadzis JM, et al: CyberKnife stereotactic radiosurgical treatment of spinal tumors for pain control and quality of life. J Neurosurg Spine 2: 540-549, 2005.

16. Schweikard A, Shiomi H and Adler J: Respiration tracking in radiosurgery. Med Phys 31: 2738-2741, 2004

17. Collins BT, Erickson K, Reichner CA, et al: Radical stereotactic radiosurgery with real-time tumor motion tracking in the treatment of small peripheral lung tumors. Radiat Oncol 2: 39 , 2007.

18. Reichner C, Collins B, Gagnon G, et al: The placement of gold fiducials for CyberKnife stereotactic radiosurgery using a modified transbronchial needle aspiration technique. J Bronchol 12: 193-195, 2005.

19. Therasse P, Arbuck SG, Eisenhauer EA, et al: New guidelines to evaluate the response to treatment in solid tumors. J Natl Cancer Inst 92: 205-216, 2000

20. Papiez L, Timmerman R, DesRosiers C, et al: Extracranial stereotactic radioablation: physical principles. Acta Oncol 42: 882-894, 2003

21. Simpson JR, Francis ME, Perez-Tamayo R, et al: Palliative radiotherapy for inoperable carcinoma of the lung: final report of a RTOG multi-institutional trial. Int J Radiat Oncol Biol Phys 11: 751-758, 1985.

22. Roh KW, Jang JS, Kim MS, et al: Fractionated stereotactic radiotherapy as reirradiation for locally recurrent head and neck cancer. Int J Radiat Oncol Biol Phys 74: 1348-1355, 2009

23. Chen HJ, Leung SW and Su CY: Linear accelerator based radiosurgery as a salvage treatment for skull base and intracranial invasion of recurrent nasopharyngeal carcinoma. J Clin Oncol 24: 255-258, 2001.
24. Sharma SC: Clinical implications of adopting Monte Carlo treatment planning for CyberKnife. J Appl Clin Med Phys 1: 170-175, 2010.

25. Brown WT, Wu X, Fayad F, et al: Application of robotic stereotactic radiotherapy to peripheral stage I nonsmall cell lung cancer with curative intent. Clin Oncol 21: 623-631, 2009.

26. Brown WT, Wu X, Wen BC, et al: Early results of CyberKnife image-guided robotic stereotactic radiosurgery for treatment of lung tumors. Comput Aided Surg 12: 253-261, 2007.

27. Errett LE, Wilson J, Chiu RC, et al: Wedge resection as an alternative procedure for peripheral bronchogenic carcinoma in poor-risk patients. J Thorac Cardiovasc Surg 90: 656-661, 1985.

28. Chang BK and Timmerman RD: Stereotactic body radiation therapy: a comprehensive review. Am J Clin Oncol 30: 637-644, 2007.

29. Hara W, Loo BW, Goffinet DR, et al: Excellent local control with stereotactic radiotherapy boost after external beam radiotherapy in patients with nasopharyngeal carcinoma. Int J Radiat Oncol Biol Phys 71: 393-400, 2008

30. Vavassori A, Jereczek-Fossa BA, Beltramo G, et al: Imageguided robotic radiosurgery as salvage therapy for locally recurrent prostate cancer after external beam irradiation: retrospective feasibility study on six cases. Tumori 96: 71-75, 2010.

31. Jereczek-Fossa BA, Fariselli L, Beltramo G, et al: Linac-based or robotic image-guided stereotactic radiotherapy for isolated lymph node recurrent prostate cancer. Radiother Oncol 93: 14-17, 2009.

32. Fowler JF, Welsh JS, Howard SP, et al: Loss of biological effect in prolonged fraction delivery. Int J Radiat Oncol Biol Phys 159: 242-249, 2004.

33. Thariat J, Li G, Angellier G, et al: Current indications and ongoing clinical trials with CyberKnife stereotactic radiotherapy in France in 2009. Bull Cancer 96: 853-864, 2009.

34. Calcerrada Daz-Santos N, Blasco Amaro JA, Cardiel GA, et al: The safety and efficacy of robotic image-guided radiosurgery system treatment for intra- and extracranial lesions: a systematic review of the literature. Radiother Oncol 89: 245-253, 2008.

35. Fuller DB, Naitoh J, Lee C, et al: Virtual HDR CyberKnife treatment for localized prostatic carcinoma: dosimetry comparison with HDR brachytherapy and preliminary clinical observations.Int J Radiat Oncol Biol Phys 70: 1588-1597, 2008.

36. Sharma SC, Ott JT, Williams JB and Dickow D: Commissioning and acceptance testing of a CyberKnife linear accelerator. J Appl Clin Med Phys 8: 2473, 2007.

37. Bucholz RD, Laycock KA and Cuff LE: CyberKnife stereotactic radiosurgery for intracranial neoplasms, with a focus on malignant tumors. Technol Cancer Res Treat 9: 541-550, 2010.

38. King CR, Brooks JD, Gill H, Pawlicki T, Cotrutz C and Presti JC Jr: Stereotactic body radiotherapy for localized prostate cancer: interim results of a prospective phase II clinical trial. Int J Radiat Oncol Biol Phys 73: 1043-1048, 2009.

39. Wowra B, Muacevic A and Tonn JC: Quality of radiosurgery for single brain metastases with respect to treatment technology: a matched-pair analysis. J Neurooncol 94: 69-77, 2009.

40. Ahmed HU, Ishaq A, Zacharakis E, et al: Rectal fistulae after salvage high-intensity focused ultrasound for recurrent prostate cancer after combined brachytherapy and external beam radiotherapy. BJU Int 103: 321-323, 2009.

41. Choi BO, Choi IB, Jang HS, et al: Stereotactic body radiation therapy with or without transarterial chemoembolization for patients with primary hepatocellular carcinoma: preliminary analysis. BMC Cancer 8: 351, 2008. 